\title{
Traditional Chinese medicine, liver fibrosis, intestinal flora: is there any connection? - a narrative review
}

\author{
Yu-Tong Liu ${ }^{1}$, Shuang-Lin $\mathrm{Qi}^{1}$, Ke-Wei Sun ${ }^{2}$ \\ ${ }^{1}$ Department of Traditional Chinese Medicine, Graduate School, Hunan University of Chinese Medicine, Changsha, China; ${ }^{2}$ Department of \\ Infection, The First Hospital of Hunan University of Chinese Medicine, Changsha, China \\ Contributions: (I) Conception and design: All authors; (II) Administrative support: None; (III) Provision of study materials or patients: None; (IV) \\ Collection and assembly of data: All authors; (V) Data analysis and interpretation: All authors; (VI) Manuscript writing: All authors; (VII) Final \\ approval of manuscript: All authors. \\ Correspondence to: Ke-Wei Sun, PhD. Professor, Department of Infection, The First Hospital of Hunan University of Chinese Medicine, No. 95, \\ Shaoshan Middle Road, Changsha 410007, China. Email: Keweisun550@163.com.
}

\begin{abstract}
This paper aims to analyze how intestinal flora regulates liver fibrosis pathogenesis and to evaluate the regulatory effect of traditional Chinese medicine (TCM) on the intestinal flora, providing new insights into liver fibrosis treatment. Destruction of the intestinal microbiome can lead to liver fibrosis development, accelerating the intestinal microbiome's disruption. TCM can effectively regulate the intestinal flora, helping prevent and treat liver fibrosis. This review discusses the mechanisms behind intestinal flora changes in liver fibrosis and how TCM can regulate these changes. We searched PubMed, the Wanfang database, and CNKI for "liver fibrosis", "intestinal microflora", and "intestinal microbiota" and reviewed the retrieved literature. We detail the prevention and treatment options for liver fibrosis though the use of TCM in regulating intestinal flora. We also highlight the influence of the intestinal flora on liver fibrosis and present the research regarding the prevention and treatment of liver fibrosis using TCM. We also describe the effects of TCM on the intestinal flora. TCM can effectively regulate the intestinal flora to prevent and treat liver fibrosis through the liver-intestine axis.
\end{abstract}

Keywords: Liver fibrosis; intestinal flora; traditional Chinese medicine (TCM); review

Submitted Oct 28, 2020. Accepted for publication Feb 25, 2021.

doi: 10.21037/apm-20-2129

View this article at: http://dx.doi.org/10.21037/apm-20-2129

\section{Introduction}

\section{Background}

The occurrence and development of liver fibrosis (Figure 1) occurs in all chronic liver diseases and leads to liver cirrhosis or even liver cancer. The mortality rate of patients with liver cirrhosis is ranked 13th among all chronic diseases, suggesting that this condition has significant effects on quality of life (1). Studies have shown that liver fibrosis and early diagnosed cirrhosis can be reversed, particularly for those who require liver fibrosis treatment (2). Liver fibrosis is caused by excessive deposition of the extracellular matrix components and the disruption of hepatic sinusoidal endothelial cell functions. Hepatic sinusoidal endothelial cells are the gatekeepers of a stable liver environment under physiological conditions. The antifibrotic effects of the sinusoidal endothelial cells prevent Kupffer cell activation and the formation of hepatic stellate cells, enabling hepatic vascular resistance regulation and reducing venous pressure (3). The capillary vascularization of these hepatic sinusoidal endothelial cells in the endothelial basement membrane forms multiple pathological factors and occurs during the early stages of liver fibrosis, thereby altering liver function (4). Disorders of the intestinal flora can also lead to liver disease occurrence and development (5). Notably, the intestine and liver are inseparable from each other 


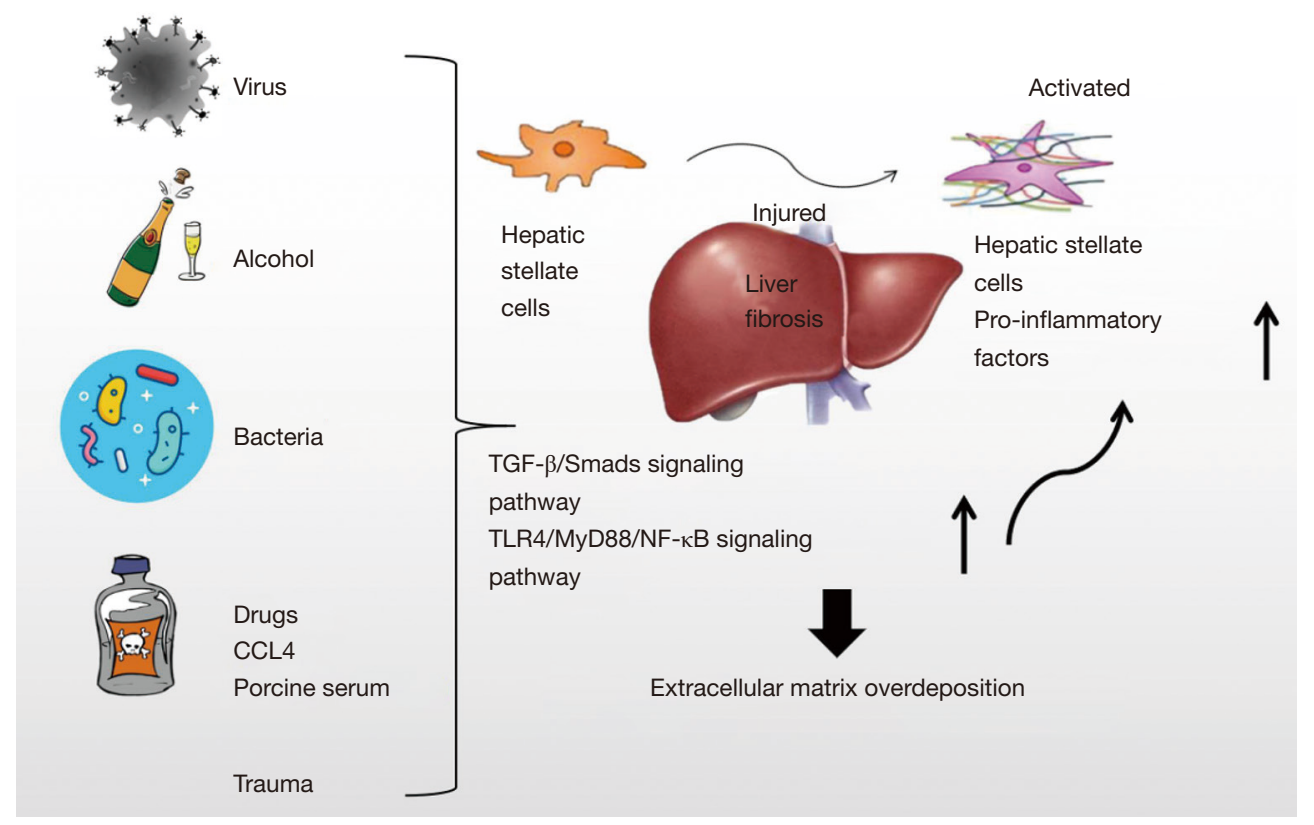

Figure 1 Pathogenesis of liver fibrosis. Induction by viruses, alcohol consumption, bacteria, trauma, and other factor leads to activation of hepatic stellate cells, deposition of the excessive extracellular matrix, and release of inflammatory factors, eventually leading to liver fibrosis.

anatomically, and the intestine supplies $75 \%$ of the blood to the hepatic portal vein (6).

Moreover, the intestinal tract and liver functionally interact, and thus homeostasis of the intestinal flora is affected by liver fibrosis (7).

One study showed that liver fibrosis could lead to disorders of the intestinal flora, and NADPH oxidase 4 and RhoA could accelerate the development of liver fibrosis by activating the hepatic stellate cells, leading to dysbacteriosis (8). Under the pathological changes induced by liver fibrosis, an increase in altered Schaedler flora of the intestine accelerates fibrosis progression; this effect can be blocked by inhibiting the altered Schaedler flora and promoting the growth of the specific pathogen-free flora instead (9).

This review discusses the liver fibrosis treatment with traditional Chinese medicine (TCM) and highlights the limitations of current research on preventing liver fibrosis by regulating the intestinal flora via TCM. We also explore new approaches for treating liver diseases by regulating the intestinal flora with TCM to provide a theoretical basis and clinical practice guide.

We present the following article in accordance with the Narrative Review reporting checklist (available at http:// dx.doi.org/10.21037/apm-20-2129).

\section{Methods}

Information on the literature review in this paper is shown in Table 1.

\section{TCM regulates intestinal flora}

\section{Astragalus}

Astragalus polysaccharide an effective extract of Astragalus membranaceus (10) and functions as an inflammatory reaction inhibitor by reducing the expression of proinflammatory factors, such as interleukin (IL)-6 and tumor necrosis factor (TNF)- $\alpha$ (11). Astragalus polysaccharides can also increase Lactobacillus and Bifidobacterium production in the intestinal tract and reduce Salmonella typhi count, which reduces inflammatory reaction occurrences (12). As a result of this reduction, Astragalus polysaccharides can exert antiinflammatory effects by regulating the numbers of bacteria (13). Additionally, A. membranaceus can alter the intestinal flora's structure and composition and enrich its diversity (14).

\section{Scutellaria baicalensis}

Scutellaria baicalensis is a traditional Chinese herbal medicine that has many beneficial effects; it is anti-inflammatory, 
Table 1 Sources for this review

1. PubMed: from the establishment of the database to October 2020, we searched for "liver fibrosis", "intestinal microflora",

"intestinal microorganisms" and "traditional Chinese medicine"

2. CNKI/Wanfang database: from the establishment of the database to October 2020, we searched for "liver fibrosis", "intestinal flora", and "traditional Chinese medicine"

3. Manual retrieval of references in literature

antibacterial, and has antioxidants properties (15). S. baicalensis can effectively promote healthy bacterial growth (Bifidobacterium and Lactobacillus) in the intestinal tract (16). Notably, Lactobacillus rbamnosus, L. paracasei, and L. plantarum can effectively reduce liver fat percentage and halt the occurrence and/or development of alcoholic liver disease (17). Additionally, Bifidobacterium and L. Rhamnosus can reduce liver inflammation and block the occurrence of nonalcoholic fatty liver disease (18).

\section{Pueraria lobata}

Pueraria lobata comes from the dried roots of leguminous plants and has been shown to have medicinal value. Pueraria polysaccharides are extracted from P. Lobata and can be consumed by intestinal microorganisms to benefit metabolism, ultimately affecting the body's physiological function $(19,20)$. After it is fermented and metabolized in the intestinal flora, this TCM can effectively improve drug properties and reduce drug toxicity (21).

P. Lobata reduces the expression of inflammatory factors, such as TNF- $\alpha$ and IL-6. The expression levels of these inflammatory factors after Bifidobacterium fermentation when using $P$. Lobata were shown to be significantly lower than those in Pueraria without fermentation (22). Also, in the small intestine and colon of mice fed with a high-fat diet, puerarin, a functional extract of $P$. Lobata, was shown to block the expression of the proinflammatory factors IL-6 and monocyte chemoattractant protein-1 and increase the expression of the anti-inflammatory factor IL-10 (23). Other studies have shown that Pueraria can regulate intestinal flora disorder (24) and prevent liver injury by reducing endotoxin contents in the intestinal tract (25).

\section{Monkshood (Fuzi)}

The TCM aconite lateral root (Fuzi) has been widely used as an anti-inflammatory drug, analgesic, diuretic, and cardiotonic to treat colds, heart failure, edema, and other diseases (26). This medicine still retains a certain degree of toxicity and can cause toxicity-induced ventricular tachycardia; however, processed aconite's toxicity is significantly reduced (27). Furthermore, experimental studies have confirmed that the content of monoester and diterpenoid alkaloids in processed Aconitum is reduced after being metabolized by the intestinal flora, resulting in lower toxicity (28). It has further been shown capable of inhibiting the pathogenicity of harmful bacteria, such as Staphylococcus aureus ATCC-25923, and exhibits antitumor effects, including the inhibition of HL-60, SMCC-7721, and SW480 tumor cell growth (29). The metabolism of this TCM after intestinal flora metabolism can be directly analyzed using real-time ionization combined with multistage tandem mass spectrometry, enabling a quantitative analysis (30). Aconite also has inhibitory effects on Escherichia coli and $S$. aureus $(31,32)$.

\section{Largehead Atractylodes rhizome}

In contrast to Western medicine, TCM places more importance on the whole body as a concept, considering patients as an organic and unified whole; thus, TCM has significant system biology advantages (33). The metabolism of intestinal microorganisms is a key factor affecting the therapeutic effects of TCMs in vivo (34).

The TCM, Atractylodes macrocephala, has the effect of invigorating the spleen, promoting dampness, and stopping diarrhea; therefore, it is used to treat diarrhea caused by spleen weakness. After treatment with $A$. macrocephala, the pathogenic bacteria, such as Helicobacter pylori and Clostridium, are significantly reduced. In contrast, beneficial bacteria, such as Lactobacillus and Akerman's bacteria, are significantly increased, indicating that $A$. macrocephala promotes probiotic reproduction and maintains the flora's intestinal homeostasis (35).

The therapeutic effects of $A$. macrocephala in vivo are related to the regulation of E. coli, ShigellaI, and Alternaria, and the intestinal flora's homeostasis (36). Also, $A$. 


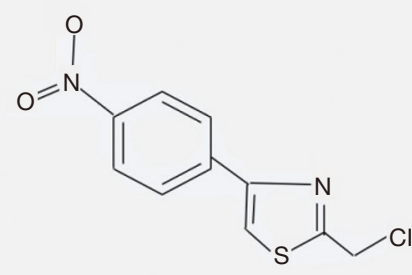

Astragalus polysaccharides
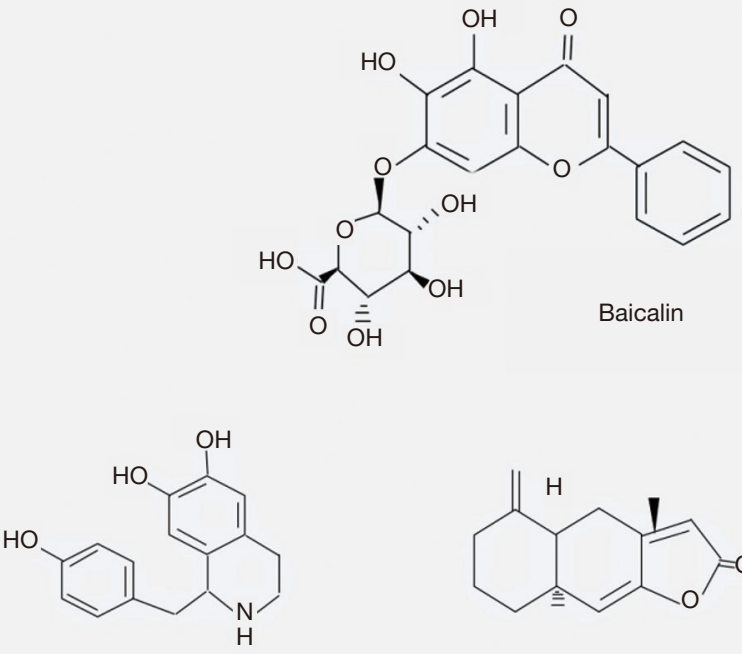

Aconitine

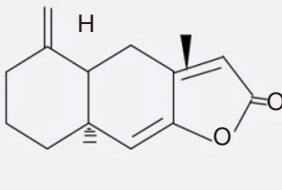

Atractylolide I

Figure 2 Organic chemical structure of the active fraction of TCM. TCM, traditional Chinese medicine.

macrocephala oil can reduce $H$. pylori biofilm formation, thereby inhibiting the proliferation of $H$. pylori and reducing the expression of the inflammatory factor IL-8 (37). A. macrocephala has also been shown to regulate intestinal microorganisms, stabilize the internal environment, and effectively alleviate chronic gastrointestinal diseases (38). $A$. macrocephala polysaccharides can also regulate disorders of the intestinal flora (39), and atractylodin can block E. coli, Bacillus subtilis, Candida albicans, and S. aureus activity $(40,41)$.

\section{Summary of the effects of TCMs on the intestinal flora}

TCM and their effective compounds (Figure 2) have long been used to treat liver fibrosis, showing remarkable curative effects (42). According to existing literature, TCMs can regulate the intestinal flora's homeostasis to prevent the occurrence and development of liver fibrosis. For example, Schisandra chinensis can protect the liver and block hepatic fibrosis by regulating intestinal microorganisms and reversing the abnormal bile acid spectrum $(43,44)$.

\section{The liver-intestine axis}

There are anatomical and physiological connections between the liver and intestines in the human body (Figure 3). The chemical signals that connect these organs make up the liver-intestine axis and include biological processes, such as metabolism and supporting of the internal environment's stability (45). Imbalances in the intestinal flora and hyperproliferation of these microorganisms can lead to the destruction of the intestinal barrier. Because the intestinal flora flows into the liver through the portal vein (46), disruptions of the intestinal flora lead to immune responses in the liver and aggravated liver fibrosis occurrence and development (47). However, homeostasis of the intestinal flora supports the stability of the immune system (48). Notably, the intestinal microflora of rats with hepatic fibrosis was found to be altered (49), and another study showed that dysfunction of the intestinal barrier and flow of intestinal microorganisms into the blood induces inflammatory reactions and eventually leads to liver injury and liver fibrosis (50). These changes also reduce the contents of bacterial metabolites, such as lysozyme and lysophosphatide, which can induce liver fibrosis and inhibit Kupffer cell activation (51).

The liver-intestine axis has a two-way regulation mechanism; that is, liver fibrosis can affect the intestinal microbiome, and the intestinal microbiome can affect liver fibrosis (52). In liver fibrosis caused by chronic hepatitis $\mathrm{B}$, the abundance of the intestinal microbiota decreases, thereby affecting bile acid metabolism (53). Ursolic acid can increase the numbers of Lactobacillus and Bifidobacterium in the flora, improve bacterial malnutrition, support the 


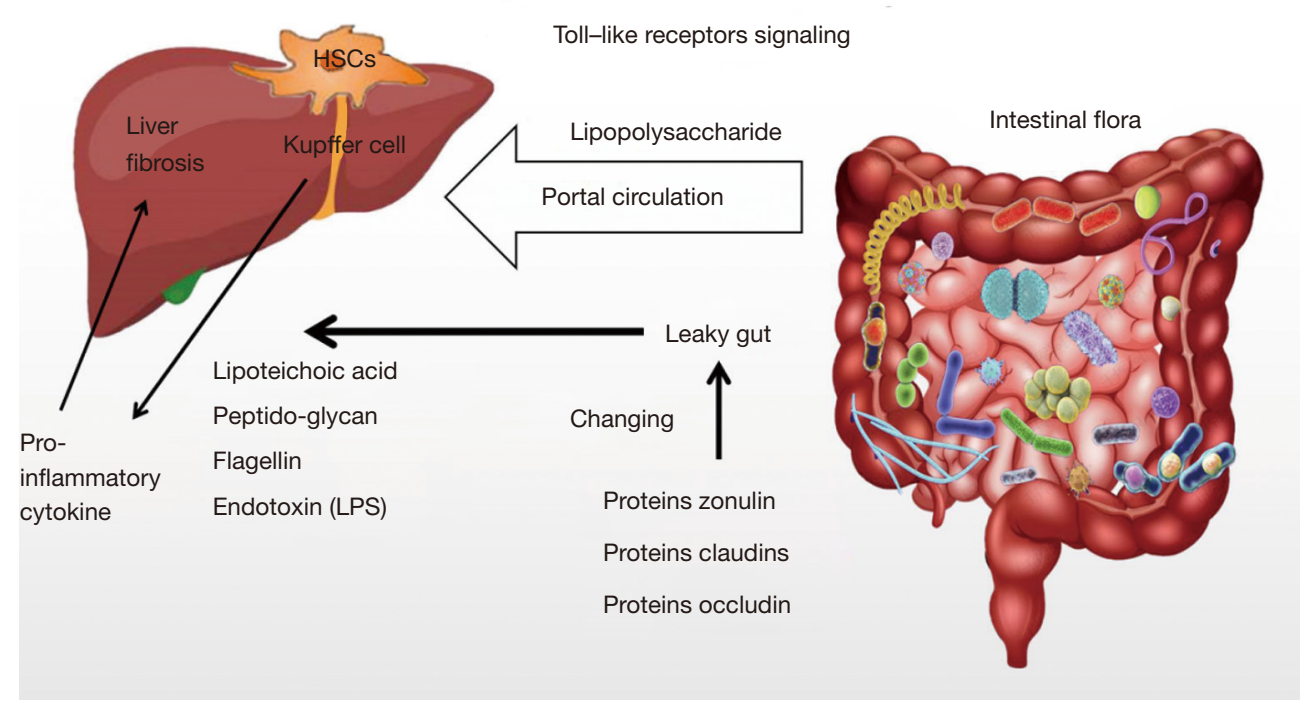

Figure 3 Pathogenic mechanisms of the intestinal flora through the liver-intestine axis. HSCs, hematopoietic stem cells.

stability of the bacterial flora, and inhibit liver fibrosis development $(54,55)$. Moreover, supplementation with the probiotic L. rhamnosus was shown to reduce liver inflammation and fibrosis (56), and the composition of the intestinal flora has been associated with inflammation and liver fibrosis (57).

\section{Summary of the liver-intestine axis}

A summary of liver-intestine axis studies is shown in Table 2. Briefly, because of various stimulating factors, intestinal wall permeability increases, and the bacteria in the intestine enter the liver through portal vein circulation, leading to liver inflammation and subsequent liver fibrosis. The results of these experimental studies (58-67) have shown that TCM extracts can reduce the degree of liver fibrosis by regulating the proportion of intestinal microorganisms, such as Firmicutes. Additionally, liver fibrosis affects the homeostasis of intestinal flora (68).

\section{Diagnosis of liver fibrosis in TCM}

TCM refers primarily to traditional medicines created by Han people in China, and involves the study of human physiology, pathology, disease diagnosis, prevention, and treatment. Herbal medicine refers to the substances used in the prevention, treatment, and diagnosis of diseases, and the rehabilitation of patients under the guidance of TCM theory. In TCM, several drugs can be combined and decocted into a soup and administered as a prescription. The prescription is typically composed of four parts: "monarch", "minister", "adjuvant", and "envoy" medicine.

Liver fibrosis is interpreted as "chest pain" and "jaundice" in TCM theory. The pathogenesis is typically stagnation of liver qi, blood stasis, and deficiency of liver yin. The pathogenesis of liver fibrosis is most commonly related to dampness, heat, phlegm, toxin, depression, blood stasis, qi deficiency (which acts on the liver meridian), and blood stasis blocking the liver vein (69).

\section{Treatment of liver fibrosis with TCM}

TCM is commonly used for the treatment of liver fibrosis (Figure 4). Studies have confirmed that Forsythia suspensa can reduce liver fibrosis progression through the transforming growth factor TGF- $\beta /$ Smad signaling pathway and the Toll-like receptor TLR 4/MyD88/nuclear factor NF- $\mathrm{KB}$ signaling pathway (70,71). Emodin, an effective rhubarb extract, can block liver fibrosis progression through the TGF- $\beta$ /Smad signaling pathway (72). Gan Shen Fu Fang can inhibit T6 hepatic stellate cell activation and block hepatic fibrosis (73). TCM has multichannel and multitarget pharmacological effects in the treatment of liver fibrosis.

Additionally, the method of syndrome differentiation and treatment in TCM has multiple advantages $(74,75)$. For example, the Dahuang Zhechong pill can inhibit the development of hepatic fibrosis by deactivating hepatic stellate cells (76). Further, A. membranaceus can inhibit 
Table 2 Changes in the intestinal flora in patients with liver fibrosis

\begin{tabular}{|c|c|c|c|}
\hline Study & Induction of liver fibrosis & Changes in the intestinal flora & Research type \\
\hline Schwimmer et al., 2019, (58) & High fat diet & Firmicutes $\uparrow$, Proteobacteria $\uparrow$ & Cross-sectional \\
\hline Lee et al., 2020, (59) & High fat diet & $\begin{array}{l}\text { Bacteroidetes } \downarrow \text {, Lachnospiraceae } \downarrow \text {, } \\
\text { Prevotellaceae } \uparrow, \text { Ruminococcaceae } \uparrow\end{array}$ & Cross-sectional \\
\hline Naito et al., 2020, (60) & High fat diet & Firmicutes $\uparrow$, Bacteroidetes $\downarrow$ & Cross-sectional \\
\hline Zhang et al., 2021, (61) & High fat diet & Bacteroidetes $\downarrow$, Firmicutes $\downarrow$ & $\mathrm{RCT}$ \\
\hline Li et al., 2020, (62) & High fat diet & $\begin{array}{l}\text { Firmicutes } \uparrow, \text { Bacteroidetes } \downarrow \text {, } \\
\text { Verrucomicrobia } \downarrow \text {, Cyanobacteria } \downarrow\end{array}$ & $\mathrm{RCT}$ \\
\hline Yan et al., 2019, (65) & CCL4 & Bacteroidetes $\downarrow$, Firmicutes $\downarrow$ & $\mathrm{RCT}$ \\
\hline Wan et al., 2019, (66) & CCL4 & Firmicutes $\downarrow$ & $\mathrm{RCT}$ \\
\hline Wu et al., 2020, (67) & CCL4 & $\begin{array}{l}\text { Proteobacteria } \downarrow \text {, Bacteroidetes } \downarrow \text {, } \\
\text { Firmicutes } \downarrow \text {, Prevotella } \uparrow\end{array}$ & $\mathrm{RCT}$ \\
\hline
\end{tabular}

The included literature shows that liver fibrosis impacts the homeostasis of intestinal flora (68). RCT, randomized controlled trial; CCL4, carbon tetrachloride.

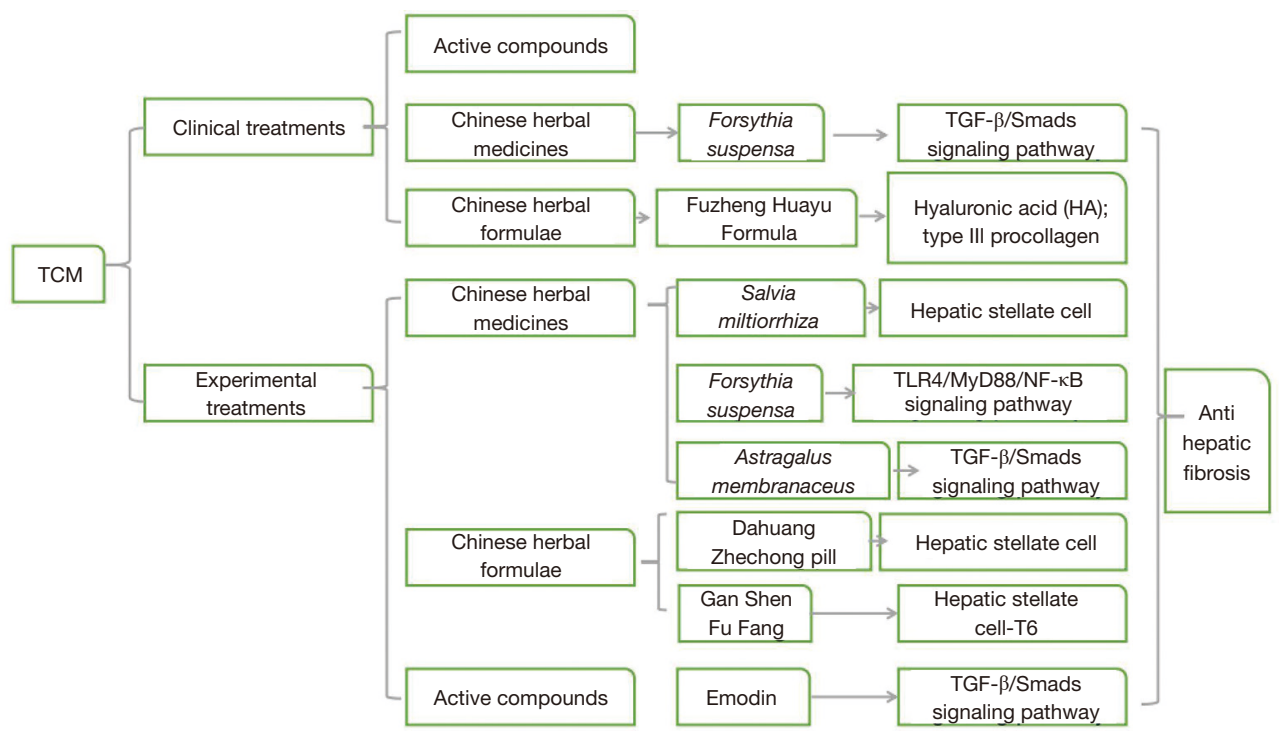

Figure 4 Effects of TCMs on liver fibrosis. The effects of TCM on liver fibrosis are described according to active compounds, Chinese herbal medicines, and Chinese herbal formulae prescription, respectively. TCM, traditional Chinese medicine. 


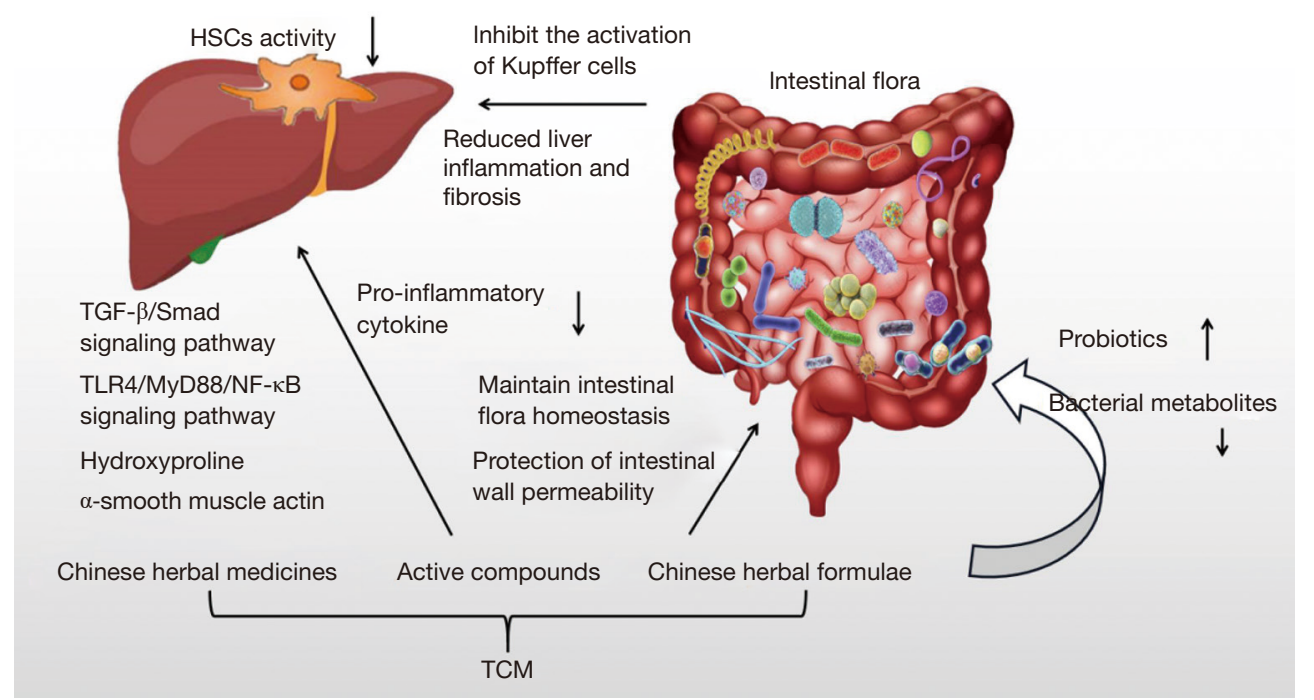

Figure 5 Potential mechanisms through which TCMs mediate the intestinal flora to protect against liver fibrosis. TCM, traditional Chinese medicine; HSCs, hematopoietic stem cells.

hepatic fibrosis by blocking the TGF- $\beta /$ Smad signaling pathway (77). Also, Sophora flavescens, Ligustrum lucidum, licorice, Lonicera japonica Thunb, and Salvia miltiorrbiza Bge are TCMs used to treat liver fibrosis (78-81). Experimental studies have shown that S. miltiorrbiza can inhibit hepatic stellate cell activity by enhancing natural killer cell functions to block liver fibrosis (82). Moreover, TCMs can protect the liver and reduce liver fibrosis by improving inflammation and reducing steatosis (83).

\section{Summary of studies of the effects of TCM on liver fibrosis}

Researchers have evaluated the effects of active compounds, Chinese herbal medicines, and Chinese herbal formulae on liver fibrosis through animal experiments and clinical studies. TCM has been shown to reduce the secretion of inflammatory factors, regulate the TGF- $\beta /$ Smad signaling pathway and TLR4/MyD88/NF- $\mathrm{BB}$ signaling pathway, inhibit the activation of hepatic stellate cells, and improve liver function, thereby blocking fibrosis and protecting the liver.

\section{Intestinal flora-related mechanisms of TCM against liver fibrosis}

TCM can effectively prevent liver fibrosis occurrence and development by regulating intestinal flora, inhibiting the intestinal barrier dysfunction, and reducing inflammatory reactions $(84,85)$. Moreover, the hepatic intestinal axis has been shown to have bidirectional regulatory effects, and TCM has been proven to protect the liver through the liver-intestine axis (86). Notably, changes in intestinal flora can induce the development of liver fibrosis and liver injury. After liver fibrosis, intestinal permeability and the fecal flora change significantly (87). Additionally, increased intestinal bacterial translocation, as a marker of chronic liver disease, can lead to hepatitis and fibrosis (88).

Berberine alters the intestinal flora of Bacteroides, xenobiotics, and Verrucosa and reduces inflammatory factor expression (89). Coptis chinensis can protect against rat liver toxicity induced by cinnabar. These mechanisms may be related to endogenous metabolism, including energy metabolism, amino acid metabolism, and intestinal flora metabolism in rats (90).

\section{Summary of the effects of TCM on liver fibrosis through the liver-intestine axis}

Studies of TCMs have evaluated these medicines' ability to protect against liver fibrosis by regulating intestinal microorganisms (Figure 5). These findings have supplied insights into novel treatment strategies and approaches for liver fibrosis. For example, the Simiao decoction can reduce inflammatory factor secretion and regulate intestinal flora homeostasis (91). Additionally, a TCM formula was found 
to reverse increases in scleroderma and Proteobacteria caused by a high-fat diet, resulting in a decreased scleroderma to Bacteroides ratio and an increased abundance of Bacteroides (92). Therefore, TCMs can effectively regulate the homeostasis of the intestinal flora, protect the intestinal wall's permeability, increase the contents of probiotics, mediate inflammatory reactions and liver lipid metabolism, block liver fibrosis, and protect the liver.

\section{Conclusions}

In this review, we presented findings of earlier studies related to the use of TCMs to regulate intestinal microorganisms and protect against liver fibrosis. However, existing research is not comprehensive, and further studies are needed. Moreover, in future works, it will be necessary to combine the holistic view of the TCM theory and methods of syndrome differentiation and treatment to explore the effects of TCMs on liver fibrosis. Additionally, use nano-imaging technology may be need to track the path of TCMs in the intestines.

\section{Acknowledgments}

We thank our team at the Hunan University of Chinese Medicine for their aid.

Funding: This study received funding from the National Natural Science Foundation of China (No. 81704068).

\section{Footnote}

Reporting Checklist: The authors have completed the Narrative Review reporting checklist. Available at http:// dx.doi.org/10.21037/apm-20-2129

Peer Review File: Available at http://dx.doi.org/10.21037/ apm-20-2129

Conflicts of Interest: All authors have completed the ICMJE uniform disclosure form (available at http://dx.doi. org/10.21037/apm-20-2129). The authors have no conflicts of interest to declare.

Ethical Statement: The authors are accountable for all aspects of the work in ensuring that questions related to the accuracy or integrity of any part of the work are appropriately investigated and resolved.

Open Access Statement: This is an Open Access article distributed in accordance with the Creative Commons Attribution-NonCommercial-NoDerivs 4.0 International License (CC BY-NC-ND 4.0), which permits the noncommercial replication and distribution of the article with the strict proviso that no changes or edits are made and the original work is properly cited (including links to both the formal publication through the relevant DOI and the license). See: https://creativecommons.org/licenses/by-nc-nd/4.0/.

\section{References}

1. Qiu BF, Zhang GQ, Xu FM, et al. Effect of the transdifferentiation of BECs into myofibroblasts on the pathogenesis of secondary cholestatic hepatic fibrosis. Exp Ther Med 2019;17:2769-76.

2. Casas-Grajales S, Reyes-Gordillo K, Cerda-GarcíaRojas CM, et al. Rebaudioside A administration prevents experimental liver fibrosis: an in vivo and in vitro study of the mechanisms of action involved. J Appl Toxicol 2019;39:1118-31.

3. Hammoutene A, Rautou PE. Role of liver sinusoidal endothelial cells in non-alcoholic fatty liver disease. J Hepatol 2019;70:1278-91.

4. Łotowska JM, Sobaniec-Łotowska ME, Sobaniec P, et al. Liver sinusoidal endothelial cells in morphogenesis of pediatric autoimmune hepatitis. Ultrastructural characteristics - a novel report. Pol J Pathol 2018;69:327-34.

5. Song Q, Zhu Z. Using Cordyceps militaris extracellular polysaccharides to prevent $\mathrm{Pb} 2+$-induced liver and kidney toxicity by activating Nrf2 signals and modulating gut microbiota. Food Funct 2020;11:9226-39.

6. Henao-Mejia J, Elinav E, Thaiss CA, et al. Role of the intestinal microbiome in liver disease. J Autoimmun 2013;46:66-73.

7. Assimakopoulos SF, Tsamandas AC, Tsiaoussis GI, et al. Altered intestinal tight junctions' expression in patients with liver cirrhosis: a pathogenetic mechanism of intestinal hyperpermeability. Eur J Clin Invest 2012;42:439-46.

8. Tao Y, Qiu T, Yao X, et al. IRE1 $\alpha /$ NOX4 signaling pathway mediates ROS-dependent activation of hepatic stellate cells in $\mathrm{NaAsO} 2$-induced liver fibrosis. J Cell Physiol 2021;236:1469-80.

9. Moghadamrad S, Hassan M, McCoy KD, et al. Attenuated fibrosis in specific pathogen-free microbiota in experimental cholestasis- and toxin-induced liver injury. FASEB J 2019;33:12464-76.

10. Zhang W, Ma W, Zhang J, et al. The immunoregulatory activities of astragalus polysaccharide liposome on 
macrophages and dendritic cells. Int J Biol Macromol 2017;105:852-61.

11. Zhao HM, Wang Y, Huang XY, et al. Astragalus polysaccharide attenuates rat experimental colitis by inducing regulatory $\mathrm{T}$ cells in intestinal Peyer's patches. World J Gastroenterol 2016;22:3175-85.

12. Bhosale M, Kadthur JC, Nandi D. Roles of Salmonella enterica serovar Typhimurium encoded Peptidase N during systemic infection of Ifn -/- mice. Immunobiology 2012;217:354-62.

13. Dong N, Li X, Xue C, et al. Astragalus polysaccharides attenuated inflammation and balanced the gut microflora in mice challenged with Salmonella typhimurium. Int Immunopharmacol 2019;74:105681.

14. Han C, Jiang YH, Li W, et al. Study on the Antihypertensive Mechanism of Astragalus membranaceus and Salvia miltiorrhiza Based on Intestinal Flora-Host Metabolism. Evid Based Complement Alternat Med 2019;2019:5418796.

15. Han YK, Kim H, Shin H, et al. Characterization of Anti-Inflammatory and Antioxidant Constituents from Scutellaria baicalensis Using LC-MS Coupled with a Bioassay Method. Molecules 2020;25:3617.

16. Shin NR, Gu N, Choi HS, et al. Combined effects of Scutellaria baicalensis with metformin on glucose tolerance of patients with type 2 diabetes via gut microbiota modulation. Am J Physiol Endocrinol Metab 2020;318:E52-61.

17. Tsai YS, Lin SW, Chen YL, et al. Effect of probiotics Lactobacillus paracasei GKS6, L. plantarum GKM3, and L. rhamnosus GKLC1 on alleviating alcohol-induced alcoholic liver disease in a mouse model. Nutr Res Pract 2020;14:299-308.

18. Wang G, Jiao T, Xu Y, et al. Bifidobacterium adolescentis and Lactobacillus rhamnosus alleviate non-alcoholic fatty liver disease induced by a high-fat, high-cholesterol diet through modulation of different gut microbiota-dependent pathways. Food Funct 2020;11:6115-27.

19. Li L, Yao H, Li X, et al. Destiny of Dendrobium officinale Polysaccharide after Oral Administration: Indigestible and Nonabsorbing, Ends in Modulating Gut Microbiota. J Agric Food Chem 2019;67:5968-77.

20. Chen R, Liu B, Wang X, et al. Effects of polysaccharide from Pueraria lobata on gut microbiota in mice. Int J Biol Macromol 2020. [Epub ahead of print]. doi:10.1016/ j.ijbiomac.2020.04.201.

21. Hussain A, Bose S, Wang JH, et al. Fermentation, a feasible strategy for enhancing bioactivity of herbal medicines. Food Res Int 2016;81:1-16.

22. Choi Y, Bose S, Shin NR, et al. Lactate-Fortified Puerariae Radix Fermented by Bifidobacterium breve Improved Diet-Induced Metabolic Dysregulation via Alteration of Gut Microbial Communities. Nutrients 2020;12:276.

23. Wang L, Wu Y, Zhuang L, et al. Puerarin prevents high-fat diet-induced obesity by enriching Akkermansia muciniphila in the gut microbiota of mice. PLoS One 2019; 14:e0218490.

24. Chen R, Wu P, Cai Z, et al. Puerariae Lobatae Radix with chuanxiong Rhizoma for treatment of cerebral ischemic stroke by remodeling gut microbiota to regulate the braingut barriers. J Nutr Biochem 2019;65:101-14.

25. Peng JH, Cui T, Sun ZL, et al. Effects of Puerariae Radix Extract on Endotoxin Receptors and TNF- Expression Induced by Gut-Derived Endotoxin in Chronic Alcoholic Liver Injury. Evid Based Complement Alternat Med 2012;2012:234987.

26. Tang L, Gong Y, Lv C, et al. Pharmacokinetics of aconitine as the targeted marker of Fuzi (Aconitum carmichaeli) following single and multiple oral administrations of Fuzi extracts in rat by UPLC/MS/MS. J Ethnopharmacol 2012;141:736-41.

27. Yang Z, Lin Y, Gao L, et al. Circadian clock regulates metabolism and toxicity of Fuzi(lateral root of Aconitum carmichaeli Debx) in mice. Phytomedicine 2020;67:153161.

28. Zhang M, Peng Y, Wang M, et al. The influence of compatibility of $\mathrm{Si}-\mathrm{Ni}$ decoction with metabolism in intestinal bacteria on transports of toxic diterpenoid alkaloids from processed aconite root across Caco-2 monolayers. J Ethnopharmacol 2019;228:164-78.

29. Liang X, Chen L, Song L, et al. Diterpenoid alkaloids from the root of Aconitum sinchiangense W. T. Wang with their antitumor and antibacterial activities. Nat Prod Res 2017;31:2016-23.

30. Li X, Hou G, Xing J, et al. Direct analysis in real timemass spectrometry for the rapid detection of metabolites of aconite alkaloids in intestinal bacteria. J Am Soc Mass Spectrom 2014;25:2181-4.

31. Lin GM, Liu L, Shao W. Microcalorimetry studies on the antibacterial effect of crude monkshood polysaccharide. J Zhejiang Univ Sci B 2011;12:563-7.

32. Zhao Y, Wang J, Sun X, et al. Microcalorimetry coupled with chemometric techniques for toxicity evaluation of Radix Aconiti Lateralis Preparata (Fuzi) and its processed products on Escherichia coli. Appl Microbiol Biotechnol 2014;98:437-44. 
33. Oberg AL, Kennedy RB, Li P, et al. Systems biology approaches to new vaccine development. Curr Opin Immunol 2011;23:436-43.

34. Harris LA, Baffy N. Modulation of the gut microbiota: a focus on treatments for irritable bowel syndrome. Postgrad Med 2017;129:872-88.

35. Shi K, Qu L, Lin X, et al. Deep-Fried Atractylodis Rhizoma Protects against Spleen Deficiency-Induced Diarrhea through Regulating Intestinal Inflammatory Response and Gut Microbiota. Int J Mol Sci 2019;21:124.

36. Ma S, Jiang Y, Zhang B, et al. Comparison of the Modulatory Effect on Intestinal Microbiota between Raw and Bran-Fried Atractylodis Rhizoma in the Rat Model of Spleen-Deficiency Syndrome. Int J Environ Res Public Health 2019;16:3183.

37. Yu M, Wang X, Ling F, et al. Atractylodes lancea volatile oils attenuated helicobacter pylori NCTC11637 growth and biofilm. Microb Pathog 2019;135:103641.

38. Inagaki N, Komatsu Y, Sasaki H, et al. Acidic polysaccharides from rhizomes of Atractylodes lancea as protective principle in Candida-lnfected mice. Planta Med 2001;67:428-31.

39. Wang R, Zhou G, Wang M, et al. The Metabolism of Polysaccharide from Atractylodes macrocephala Koidz and Its Effect on Intestinal Microflora. Evid Based Complement Alternat Med 2014;2014:926381.

40. Chen $\mathrm{Y}, \mathrm{Wu} \mathrm{Y}$, Wang H, et al. A new 9-nor-atractylodin from Atractylodes lancea and the antibacterial activity of the atractylodin derivatives. Fitoterapia 2012;83:199-203.

41. Jeong SI, Kim SY, Kim SJ, et al. Antibacterial activity of phytochemicals isolated from Atractylodes japonica against methicillin-resistant Staphylococcus aureus. Molecules 2010;15:7395-402.

42. Liao X, Bu Y, Jia Q. Traditional Chinese medicine as supportive care for the management of liver cancer: Past, present, and future. Genes Dis 2019;7:370-9.

43. Li DS, Huang QF, Guan LH, et al. Targeted bile acids and gut microbiome profiles reveal the hepato-protective effect of WZ tablet (Schisandra sphenanthera extract) against LCA-induced cholestasis. Chin J Nat Med 2020;18:211-8.

44. Hong M, Zhang Y, Li S, et al. A Network PharmacologyBased Study on the Hepatoprotective Effect of Fructus Schisandrae. Molecules 2017;22:1617.

45. Jeon JW, Choi N, Lee SH, et al. Three-tissue microphysiological system for studying inflammatory responses in gut-liver Axis. Biomed Microdevices 2020;22:65.

46. Ghoshal UC, Shukla R, Ghoshal U. Small Intestinal
Bacterial Overgrowth and Irritable Bowel Syndrome: A Bridge between Functional Organic Dichotomy. Gut Liver 2017;11:196-208.

47. Qin T, Fu J, Verkade HJ. The role of the gut microbiome in graft fibrosis after pediatric liver transplantation. Hum Genet 2021;140:709-24.

48. Ichinohe T, Pang IK, Kumamoto Y, et al. Microbiota regulates immune defense against respiratory tract influenza A virus infection. Proc Natl Acad Sci U S A 2011;108:5354-9.

49. Li Z, Ni M, Yu H, et al. Gut Microbiota and Liver Fibrosis: One Potential Biomarker for Predicting Liver Fibrosis. Biomed Res Int 2020;2020:3905130.

50. Chopyk DM, Grakoui A. Contribution of the Intestinal Microbiome and Gut Barrier to Hepatic Disorders. Gastroenterology 2020;159:849-63.

51. Tan Y, Li Y, Zhou F, et al. Administration of a mixture of triterpenoids from yeyachun and phenolic acids from danshen ameliorates carbon tetrachloride-induced liver fibrosis in mice by the regulation of intestinal flora. J Pharmacol Sci 2020;143:165-75.

52. Shah A, Macdonald GA, Morrison M, et al. Targeting the Gut Microbiome as a Treatment for Primary Sclerosing Cholangitis: A Conceptional Framework. Am J Gastroenterol 2020;115:814-22.

53. Wang X, Chen L, Wang H, et al. Modulation of bile acid profile by gut microbiota in chronic hepatitis B. J Cell Mol Med 2020;24:2573-81.

54. Ma XY, Zhang M, Fang G, et al. Ursolic acid reduces hepatocellular apoptosis and alleviates alcohol-induced liver injury via irreversible inhibition of CASP3 in vivo. Acta Pharmacol Sin 2020. [Epub ahead of print]. doi:10.1038/s41401-020-00534-y.

55. Chen D, Le TH, Shahidipour H, et al. The Role of GutDerived Microbial Antigens on Liver Fibrosis Initiation and Progression. Cells 2019;8:1324.

56. Liu Y, Chen K, Li F, et al. Probiotic Lactobacillus rhamnosus GG Prevents Liver Fibrosis Through Inhibiting Hepatic Bile Acid Synthesis and Enhancing Bile Acid Excretion in Mice. Hepatology 2020;71:2050-66.

57. Jayakumar S, Loomba R. Review article: emerging role of the gut microbiome in the progression of nonalcoholic fatty liver disease and potential therapeutic implications. Aliment Pharmacol Ther 2019;50:144-58.

58. Schwimmer JB, Johnson JS, Angeles JE, et al. Microbiome Signatures Associated With Steatohepatitis and Moderate to Severe Fibrosis in Children With Nonalcoholic Fatty Liver Disease. Gastroenterology 2019;157:1109-22. 
59. Lee G, You HJ, Bajaj JS, et al. Distinct signatures of gut microbiome and metabolites associated with significant fibrosis in non-obese NAFLD. Nat Commun 2020;11:4982.

60. Naito Y, Ushiroda C, Mizushima K, et al. Epigallocatechin-3-gallate (EGCG) attenuates nonalcoholic fatty liver disease via modulating the interaction between gut microbiota and bile acids. J Clin Biochem Nutr 2020;67:2-9.

61. Zhang X, Coker OO, Chu ES, et al. Dietary cholesterol drives fatty liver-associated liver cancer by modulating gut microbiota and metabolites. Gut 2021;70:761-74.

62. Li P, Yan K, Chang X, et al. Sex-specific maternal calcium requirements for the prevention of nonalcoholic fatty liver disease by altering the intestinal microbiota and lipid metabolism in the high-fat-diet-fed offspring mice. Gut Microbes 2020;11:1590-607.

63. Li MM, Zhou Y, Zuo L, et al. Dietary fiber regulates intestinal flora and suppresses liver and systemic inflammation to alleviate liver fibrosis in mice. Nutrition 2021;81:110959.

64. Wan S, Nie Y, Zhang Y, et al. Gut Microbial Dysbiosis Is Associated with Profibrotic Factors in Liver Fibrosis Mice. Front Cell Infect Microbiol 2020;10:18.

65. Yan Z, Yang F, Hong Z, et al. Blueberry Attenuates Liver Fibrosis, Protects Intestinal Epithelial Barrier, and Maintains Gut Microbiota Homeostasis. Can J Gastroenterol Hepatol 2019;2019:5236149.

66. Wan SZ, Liu C, Huang CK, et al. Ursolic Acid Improves Intestinal Damage and Bacterial Dysbiosis in Liver Fibrosis Mice. Front Pharmacol 2019;10:1321.

67. Wu P, Zhang R, Luo M, et al. Liver Injury Impaired 25-Hydroxylation of Vitamin D Suppresses Intestinal Paneth Cell defensins, leading to Gut Dysbiosis and Liver Fibrogenesis. Am J Physiol Gastrointest Liver Physiol 2020;319:G685-95.

68. Di Ciaula A, Baj J, Garruti G, et al. Liver Steatosis, GutLiver Axis, Microbiome and Environmental Factors. A Never-Ending Bidirectional Cross-Talk. J Clin Med 2020;9:2648.

69. Liu Y'T, Lv WL. Study on the mechanism of Qizhu granule in anti-hepatic fibrosis. Liaoning Journal of Traditional Chinese Medicine 2019;46:2092-4.

70. Fukui H. Leaky Gut and Gut-Liver Axis in Liver Cirrhosis: Clinical Studies Update. Gut Liver 2020. [Epub ahead of print]. doi:10.5009/gnl20032.

71. Hu N, Guo C, Dai X, et al. Forsythiae Fructuse water extract attenuates liver fibrosis via TLR4/MyD88/NF- B and TGF- $\beta$ /smads signaling pathways. J Ethnopharmacol 2020;262:113275.

72. Hu N, Liu J, Xue X, et al. The effect of emodin on liver disease -- comprehensive advances in molecular mechanisms. Eur J Pharmacol 2020;882:173269.

73. Du QH, Zhang CJ, Li WH, et al. Gan Shen Fu Fang ameliorates liver fibrosis in vitro and in vivo by inhibiting the inflammatory response and extracellular signalregulated kinase phosphorylation. World J Gastroenterol 2020;26:2810-20.

74. $\mathrm{Li} \mathrm{H}$. Advances in anti hepatic fibrotic therapy with Traditional Chinese Medicine herbal formula. J Ethnopharmacol 2020;251:112442.

75. El-Tantawy WH, Temraz A. Anti-fibrotic activity of natural products, herbal extracts and nutritional components for prevention of liver fibrosis: review. Arch Physiol Biochem 2019. [Epub ahead of print]. doi: 10.1080/13813455.2019.1684952.

76. Gong Z, Lin J, Zheng J, et al. Dahuang Zhechong pill attenuates CCl4-induced rat liver fibrosis via the PI3K-Akt signaling pathway. J Cell Biochem 2020;121:1431-40.

77. Wang D, Li R, Wei S, et al. Metabolomics combined with network pharmacology exploration reveals the modulatory properties of Astragali Radix extract in the treatment of liver fibrosis. Chin Med 2019;28;14:30.

78. Chu S, Zhang H, Ding L. Efficiency of Sophora flavescens-Fructus Ligustri Lucidi Drug Pairs in the Treatment of Liver Fibrosis Based on the Response Surface Method. Evid Based Complement Alternat Med 2019;2019:8609490.

79. Li X, Sun R, Liu R. Natural products in licorice for the therapy of liver diseases: Progress and future opportunities. Pharmacol Res 2019;144:210-26.

80. Miao H, Zhang Y, Huang Z, et al. Lonicera japonica Attenuates Carbon Tetrachloride-Induced Liver Fibrosis in Mice: Molecular Mechanisms of Action. Am J Chin Med 2019;47:351-67.

81. Shi MJ, Dong BS, Yang WN, et al. Preventive and therapeutic role of Tanshinone IIA in hepatology. Biomed Pharmacother 2019;112:108676.

82. Peng Y, Yang T, Huang K, et al. Salvia Miltiorrhiza Ameliorates Liver Fibrosis by Activating Hepatic Natural Killer Cells in Vivo and in Vitro. Front Pharmacol 2018;9:762.

83. Singh M, Hussain T, Firdous H, et al. Preclinical Hepatoprotective Effect of Herbalism Against Ethanol Induced Hepatotoxicity: A Review. Curr Drug Metab 2018;19:1002-11. 
84. Wang X, Shi L, Wang X, et al. MDG-1, an Ophiopogon polysaccharide, restrains process of non-alcoholic fatty liver disease via modulating the gut-liver axis. Int J Biol Macromol 2019;141:1013-21.

85. Li N, Wang B, Wu Y, et al. Modification effects of SanWei GanJiang Powder on liver and intestinal damage through reversing bile acid homeostasis. Biomed Pharmacother 2019;116:109044.

86. Ansari A, Bose S, Lim SK, et al. Combination of Scutellaria baicalensis and Metformin Ameliorates DietInduced Metabolic Dysregulation in Mice via the GutLiver-Brain Axis. Am J Chin Med 2020;48:1409-33.

87. D'Argenio G, Cariello R, Tuccillo C, et al. Symbiotic formulation in experimentally induced liver fibrosis in rats: intestinal microbiota as a key point to treat liver damage? Liver Int 2013;33:687-97.

88. Darnaud M, Faivre J, Moniaux N. Targeting gut flora to prevent progression of hepatocellular carcinoma. J Hepatol

Cite this article as: Liu YT, Qi SL, Sun KW. Traditional Chinese medicine, liver fibrosis, intestinal flora: is there any connection? — a narrative review. Ann Palliat Med 2021;10(4):48464857. doi: 10.21037/apm-20-2129
2013;58:385-7.

89. Li L, Cui H, Li T, et al. Synergistic Effect of BerberineBased Chinese Medicine Assembled Nanostructures on Diarrhea-Predominant Irritable Bowel Syndrome In Vivo. Front Pharmacol 2020;11:1210.

90. Su G, Wang H, Gao Y, et al. ${ }^{1}$ H-NMR-Based Metabonomics of the Protective Effect of Coptis chinensis and Berberine on Cinnabar-Induced Hepatotoxicity and Nephrotoxicity in Rats. Molecules 2017;22:1855.

91. Lin X, Shao T, Huang L, et al. Simiao Decoction Alleviates Gouty Arthritis by Modulating Proinflammatory Cytokines and the Gut Ecosystem. Front Pharmacol 2020;11:955.

92. Gong S, Ye T, Wang M, et al. Traditional Chinese Medicine Formula Kang Shuai Lao Pian Improves Obesity, Gut Dysbiosis, and Fecal Metabolic Disorders in High-Fat Diet-Fed Mice. Front Pharmacol 2020;11:297. 